\title{
My NIDCAP Journey as an Interpreter
}

Michiko Doi, BA

Freelance Japanese - English Conference Interpreter

\section{Introduction}

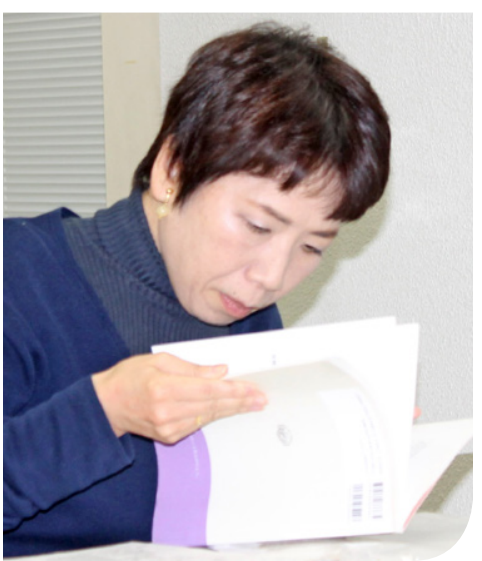

Michiko Doi

Thirteen years ago I had the opportunity to go to Tokyo, Japan with Heidelise Als to participate in a special seminar on developmental care. We were introduced to Michiko Doi who not only provided the professional translation of our lectures, but remained with us throughout our entire stay providing both interpreting and cultural guidance as needed. Little did I realize the lifelong professional and personal relationship that would evolve between us.

Michiko provides her insight into some of the difficulties for respected and experienced neonatal professionals to absorb new perspectives as NIDCAP trainees. She has demonstrated enormous patience as she interprets our complex discussions on some of the very subtle and nuanced aspects of caring for infants and families. As Michiko states, there is often more than mere translation required to communicate many of our NIDCAP terms and concepts. Michiko is the most flexible yet sturdy bidirectional bridge for our NIDCAP training communication. This involves both language and its inseparable cultural contexts. She has been indefatigable in her support of me from early mornings through late evenings, including professional work and social gatherings, from my arrival in Japan until my departure year after year.

Not only has Michiko provided her excellent service as an interpreter for the NIDCAP work in Japan, she has become the informal national advisor and counsellor as well as champion of all those involved in this work. While I have had the privilege and joy to be the NIDCAP Master Trainer for Japan articulating the voice of the newborn, Michiko has been my Japanese voice, for which I am most appreciative of her time, patience and trust.

gretchen Lawhon, PhD, RN, FAAN

Clinical Nurse Scientist with Newborn special care associates, pc at Abington - Jefferson Health NIDCAP Master Trainer with West Coast NIDCAP and APIB Training Center

A s I begin to write this article, I can't help but think of all the healthcare professionals around the world who are in the midst of the very difficult fight against the new coronavirus. They have their own families and friends as well as themselves to care for, and yet they are giving all they can to save people afflicted. My sincerest gratitude and respect goes to them.

Their dedication, however, is not only in times of emergency. It is constant - so constant that people may take it for granted. Childbirth also tends to be taken for granted. It is regarded as something that just happens, often without any problems. Now I know that it is not, especially when a baby is born early for some reason, and that is when everybody involved strives to provide the best possible care in their respective capacities. My experience as an interpreter in the NIDCAP trainings in Japan has taught me this.

I am not in the healthcare field. I am a Japanese-English interpreter by profession. Recently, in response to the growing need of international interactions in healthcare, I have often been asked to serve in healthcare-related conferences and technical visits. The NICU, however, had been an unknown world to me until I first stepped into one in 2007.

What I am about to share with you are the observations of a complete outsider. This outsider, however, has a keen interest in people, especially in a growing child as a biological and social being. So I knew even before actually becoming involved in the NIDCAP training as an interpreter that I was going to enjoy it. My encounter with NIDCAP and the journey which followed have been a truly rewarding and inspiring experience for me.

\section{The Beginning}

It was in Nagasaki in 2004 that I met Dr Akiyama, then a professor of Orthopaedic Surgery at Nagasaki University Hospital, when he organized a conference on early developmental intervention for high-risk newborns. As is always the case, I asked for relevant information such as the speakers' CVs, their presentation materials and recommended literature. These are 


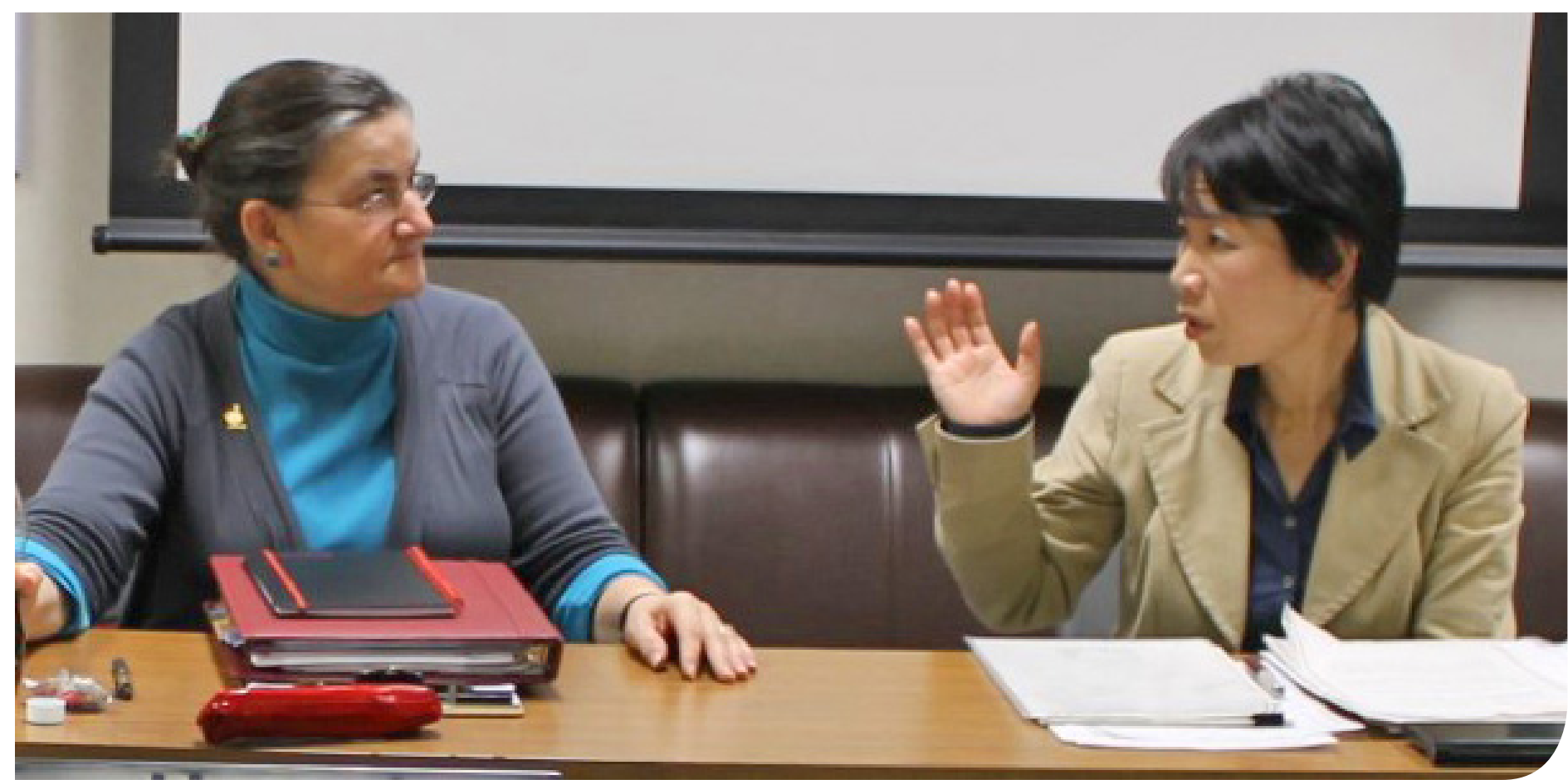

gretchen Lawhon and Michiko Doi.

very important for interpreters to make good preparation for the day. I usually start by grasping general ideas of the topic through reading all information available, then familiarise myself with the terms and expressions in both Japanese and English, and also do some research to deepen my own understanding of the topic. In the process, I came across some key concepts and terms, and among them was the name 'T. Berry Brazelton'. It turned out to be quite a challenge for me mainly because the information available in Japanese was rather limited. With the kind help of Dr Akiyama and his colleagues I survived.

In July of 2007 an opportunity presented itself to translate at a seminar with Dr Heidelise Als and Dr gretchen Lawhon at Tokyo Women's Medical University Hospital. This offer came from Dr Ohgi, once a collaborator with Dr Akiyama and then a professor of physical therapy at St Christopher's University in Hamamatsu. It was a two-day seminar including both lectures and demonstrations, which, in fact, became the first milestone for NIDCAP in Japan.

I remember the room was packed with an enthusiastic audience and filled with expectations for something innovative. Dr Nishida, then a professor of neonatology and the organiser of the seminar, later confessed he himself had little idea about what to expect because NIDCAP, at least then, was largely unknown in Japan. Therefore, there was almost no relevant information available in Japanese. All I had with me was the power point presentations prepared by the speakers, which had kindly been provided well in advance. I studied them very hard. Dr Als and Dr Lawhon were kind enough to answer the many questions I had to ask for clarification.

The fruit of this seminar was evident; it generated a momentum to move forward with NIDCAP.

\section{As a Companion}

In 2009, the first NIDCAP professional training began at Tokyo Metropolitan Hospital, Bokuto, for four trainees with gretchen. I don't think I need to go over the process here. So, instead, I would like to share some of my experience as an interpreter during the training.

The interpreter is supposed to help fill the gaps, or become a bridge in communication. Like any two languages, Japanese and English are different from each other in many ways. I usually find technical terms are easier to cope with because they usually have a one-on-one match in any two languages, so the interpreter's work is mainly to memorise. The NIDCAP terms and expressions, however, have more nuances. You really have to think what word or expression is most suitable in a given context, especially knowing that your translation will probably be used for some time in the future. Some terms in the Observation Sheet needed more than translation. We even asked gretchen to physically demonstrate them so that we could clearly grasp the link between the Japanese translation of the terms and their meanings!

Communication doesn't rely only on languages. Perfect translation doesn't always guarantee quick communication. In the training, professional backgrounds play a big part. Experienced, caring trainers like gretchen and Joy Browne have wide and profound insight into gaps arising from different backgrounds and they are prepared. The trainees, on the other hand, are likely to be trapped by such gaps, especially when they are trying to see something familiar to them in the different light. Of course, they have good reason to be. They struggle because their knowledge and experience interfere with the absorption of new perspectives. This creates a gap. 
There are also gaps stemming from differences in various systems in society including healthcare. With the word 'NICU', for instance, the picture that you may draw in your mind may be quite different from someone else's. What exists in one country or hospital may not exist in another; what is possible in one country or hospital may not be so in another. Gaps can be much more complex. So we have to be conscious of the possibility of such gaps and work towards a common understanding, and the interpreter walks the process as a companion. The beauty of all this in the training is that often this kind of struggle leads to a better understanding of the subject, and more importantly it nurtures a better relationship between the trainees and the trainer, among the trainees themselves and among all involved including the interpreter. It requires time, patience and trust in what you do regardless of the roles. I have witnessed many moments of such connectedness, and I am always impressed and supported by the nurturing attitude which dominates the team.

Currently in Japan there are nineteen NIDCAP Professionals and four are in training, involving seven hospitals. Five are in training to become trainers. How exciting!

\section{Thoughts on Universal Values in NIDCAP}

Much of my NIDCAP experience has been new to me, but I've never felt anything about it remote or irrelevant. NIDCAP, at its core, has some universal values which all humans can relate to and benefit from. Let me mention just a few.

Observation may appear to be a passive activity, but it is not. Fully committed observation like in NIDCAP can reveal so much so deeply. It is an active listening. It is from there that we gain an understanding and develop ideas and thoughts and eventually translate them into action. Reflection is another form of observation, that is, to observe your own thoughts and feelings, which I think is very important for everyone.

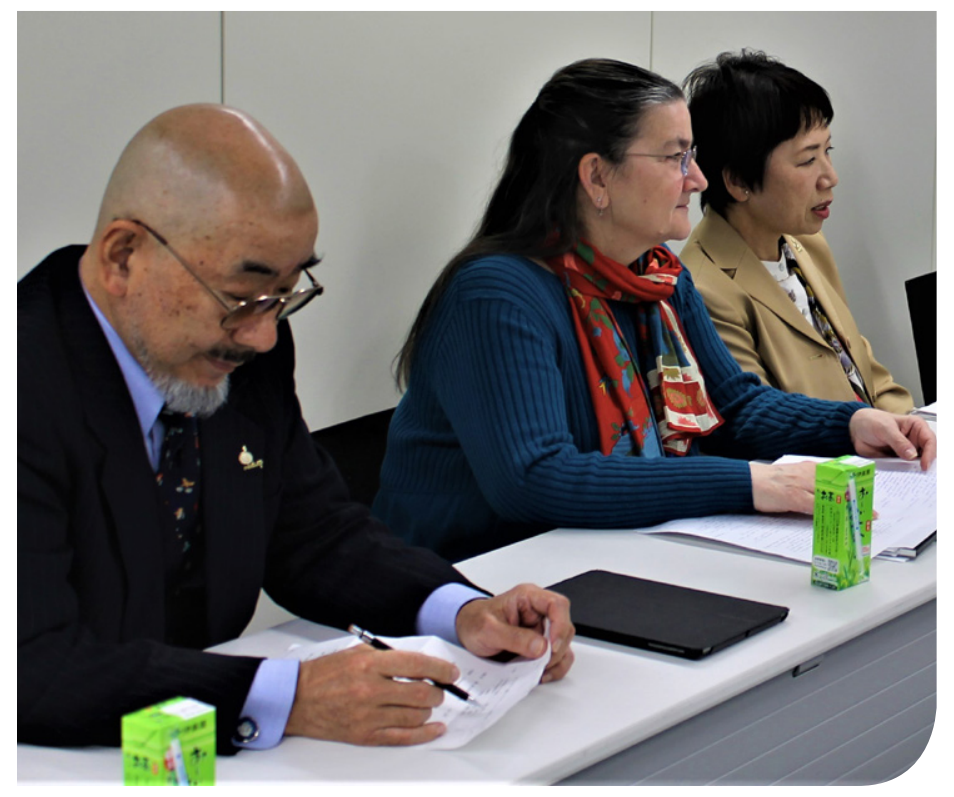

Dr Hiroshi Nishida, Dr gretchen Lawhon and Ms Michiko Doi.
In some cultures like Japanese culture, correcting weaknesses is more emphasized than acknowledging strengths. NIDCAP tells us we all have strengths to build upon from which to grow, however small we may be. 'Small' in this case can refer to the physical or to ability and capability. NIDCAP also reminds us that we grow in relationships.

Subtlety is another characteristic of NIDCAP. As an interpreter, I always need to be conscious of it and have solutions. Whenever I am tempted to think that there should be an easier way of describing something, I quickly remind myself that simplification in words may result in the dilution, if not loss, of the real meaning. While languages can open up so much space for positive communication, they also have limits when they are used just as signs and labelling for convenience. In this sense, the interpreter is just a bridge, and it is all up to the people on both ends whether they walk cross it to meet or not. Of course, I, as an interpreter, want to be a good strong bridge.

\section{Towards an End and Beyond}

In Japan, the training of NIDCAP trainers is underway and we are hoping to see the first Japanese NIDCAP trainers certified within the year. A national training centre with a multihospital structure is also in development. When all this has been achieved, my work as an interpreter will end. Sad personally, but for Japan it's really a huge celebration for the babies and their families, NICU staff, hospitals and society at large.

Dr Nishida, the honorary chairperson of Japan Developmental Care Research Association (JDCRA), has long been the principal author of 'Scientific Basis of Clinical Neonatology', one of the most valued and widely used textbooks in the field in Japan. A very important section was added to the latest 2018 edition: synactive theory and NIDCAP. It is brief but extremely significant because it is read by not only medical students but also students in nursing and other healthcare disciplines. They may not see its significance right away, but at least they know there is such an approach. The section concludes with the following remark:

"...developmental care skills and knowledge integrating NIDCAP as a pivot will be put into practice more widely in (Japanese) NICUs in the near future."

Remarks made by a Japanese neonatologist left a deep impression on me. He said, "Our unit has achieved the country's best survival rate for some years. We are proud of this, but we know it is not enough. We cannot say, 'You are fine and ready to go home, so good-bye,' when we know their lives will go on much longer. NIDCAP may provide us with a clue as to what action to take to achieve a better prognosis."

The JDCRA, the engine of NIDCAP in Japan, has updated its Website. Although most of it is in Japanese, you may still get a feel for what's going on in this part of the world.

I feel I have left out so many important people who deserve much credit in building the initial foundations of NIDCAP in Japan. I can only hope that the readers will have the opportunity to meet at least some of them in future NFI meetings. 